\title{
The Relationship Between Absence Coronary Artery Calcification and Myocardial Perfusion Single Photon Emission Computed Tomography
}

\author{
Muhannad Alanazi ${ }^{\mathrm{a}}$, Alaa AlDuraibi ${ }^{\mathrm{a}}$, Mohamamed M Shoukri ${ }^{\mathrm{b}}$, \\ Ahmed Fathala ${ }^{a, c}$
}

\begin{abstract}
Background: Coronary artery calcification score (CACS) is well validated prognostic tool in coronary artery disease (CAD). The data on the prevalence of myocardial ischemia on myocardial perfusion single photon emission computed tomography (MPS) in symptomatic patients with zero CACS and low to intermediate risk probability is lacking and controversial. The aim of our study was to evaluate the capability of zero CACS to exclude myocardial ischemia on MPS.
\end{abstract}

Methods: A total of 157 patients ((mean age $53 \pm 10$ years), 88 (56\%) female patients, 69 (44\%) male patients) who were suspected to have CAD and having low to intermediate pretest likelihood for CAD underwent CACS on dedicated computed tomography (CT) scanners. CACS was reported as zero in all patients, subsequently all patients underwent MPS. Patients with abnormal MPS underwent additional imaging with coronary computed tomography angiography (CCTA).

Results: All patients had zero CACS, of which 122 (78\%) had normal MPS, and 35 (22\%) had abnormal MPS. Abnormal MPS included fixed defect in $22(13 \%)$, equivocal in $10(6 \%)$, and reversible defect in four $(3 \%)$ patients. All patients with abnormal MPS had further imaging with CTCA. CTCA was normal in 30 (85\%) patients, one patient had coronary artery stenosis more than $50 \%$, one patient had coronary artery stenosis less than $50 \%$, one patient had anomalous origin coronary artery, and two patients had myocardial bridging. Patients with abnormal MPS and normal coronary artery had dilated cardiomyopathy in 14 (40\%), asymmetrical septal hypotrophy in one $(3 \%)$, and mitral valve disease in three $(9 \%)$.

Conclusions: Zero CACS in stable patients with low or intermediate

Manuscript submitted December 17, 2017, accepted January 4, 2018

a Department of Radiology, Nuclear Medicine and Cardiovascular Imaging, Riyadh, Saudi Arabia

${ }^{b}$ Research Center, King Faisal Specialist Hospital and Research Center, P.O. Box 3354, Riyadh, Saudi Arabia

${ }^{\mathrm{c} C}$ Corresponding Author: Ahmed Fathala, Medical Imaging Service/Department of Radiology, King Faisal Specialist Hospital and Research Center, MBC 28, P.O. Box 3354, Riyadh, Saudi Arabia. Email: ahm35799@hotmail.com

doi: https://doi.org/10.14740/cr659w risk indicated very low likelihood of obstructive CAD, less than $1 \%$. Patients with zero CACS and normal MPS most likely will not benefit from further testing; however, patients with abnormal MPS will need further imaging with CCTA. CCTA is helpful in this group of patients for evaluation of coronary artery and cardiac morphology.

Keywords: Myocardial ischemia; Cardiac SPECT; CAD; CACS

\section{Introduction}

Coronary artery calcification score (CACS) is well validated prognostic tool in coronary artery disease (CAD). There is marked increased risk of cardiovascular event and death as the CACS increases in asymptotic patients [1]. Coronary artery calcification (CAC) is an early marker of atherosclerosis and usually precedes the development of CAD by many years [2]. $\mathrm{CAC}$ was first introduced into medicine as a screening test for the presence of atherosclerosis, based on studies that demonstrated the test is a specific albeit indirect, indicator for magnitude of underling atherosclerosis [3, 4]. Most frequently, the Agatston score has been used to measure the total coronary calcified plaques [5]. Absence of calcium in the coronary arteries does not mean absence of atherosclerosis, as there may be non-calcified plaques. However, this situation correlates to a disease of lower severity [6]. In a previous study, in the symptomatic patients, the absence of measurable CAC had a high negative predictive value of obstructive CAD [7], and may exclude inducible ischemia at functional testing as myocardial perfusion positron emission tomography (PET) in patients at low or intermediate risk for CAD [8]. However, there are some studies questioned the capability of CAC to rule out the obstructive CAD. In these studies, higher rates of obstructive noncalcified plaques (6\% to $39 \%$ ) or inducible ischemia $(16 \%)$, especially in higher risk patients, unstable patients with unstable chest pain, and patients presented to the emergency department were found [9-12]. In general, the data on the prevalence of myocardial ischemia on single photon emission computed tomography (SPECT) in symptomatic patients with zero CACS and low to intermediate risk probability is lacking and controversial to some extent. Therefore, the aim of our study is to evaluate the capability of zero CACS to exclude 
myocardial ischemia on myocardial perfusion single photon emission computed tomography (MPS), and further to correlate between abnormal MPS and coronary computed tomography angiography (CCTA).

\section{Materials and Methods}

\section{Study population}

From December 2011 to January 2016, a total of 157 patients (mean age: $53 \pm 10$ years, $88(56 \%)$ female patients, $69(44 \%)$ male patients) who were suspected to have CAD and having low to intermediate pretest likelihood for CAD according to Diamond and Forrester criteria [13], underwent CACS on dedicated CT scanners. CACS was reported as zero in all patients. Subsequently all patients underwent MPS, and was reported as normal, fixed defect, equivocal, and ischemic or reversible defect. Patients with abnormal MPS underwent additional imaging with CCTA. No further workup was performed for patients with normal MPS. The study was approved by the Institutional Review Committee of the hospital.

\section{MPS acquisition and analysis}

Patients underwent rest-stress myocardial perfusion studies with either a separate day protocol or a same day stress-rest sequence. The choice of tracer and same day or separate day protocol was based on logistic requirements. The rest dose in patients who underwent a separate day rest-stress protocol was 1,100 Megabecqurel (MBq) of either technetium-99 (Tc-99m) sestamibi or tetrofosmin. The stress dose in patients who underwent the rest-stress same day protocol was 1,100 $\mathrm{MBq} \mathrm{mCi}$ of either (Tc99m) sestamibi or tetrofosmin. Tc-99m sestamibi or Tc-99m tetrofosmin was injected during peak pharmacological vasodilatation with adenosine $(140 \mu \mathrm{g} / \mathrm{kg} /$ min), or dipyridamole. SPECT imaging was started $30 \mathrm{~min}$ following pharmacological vasodilatation. Rest SPECT myocardial perfusion imaging was initiated at approximately 60 min following injection. SPECT imaging was performed with line source attenuation correction at $90^{\circ}$ dual-detector gamma camera (Cardio MD, Philips Medical System, Milpitas, California) equipped with attenuation correction and truncation compensation. The acquisition parameters and post processing were performed according to the most recent guidelines of the ASNC for nuclear cardiology procedures [14].

All images were reoriented in short, vertical, and horizontal views utilizing auto SPECT (Cedars-Sinai Medical Center, Los Angeles, California) for visual interpretation by an experienced nuclear medicine physician. The reader was not biased by clinical information. Stress and rest perfusion images were scored using 17 tomographic segments, which included six segments each for the basal and midventricular slices, and four segments for the apical short-axis slices. The final segment is located on the most apical part of the left ventricle. Finally, gated short-axis images were processed with quantitative SPECT software to measure the ejection fraction. In the visual analysis the 17 segments were scored for perfusion defects on a 4 -point system $(0=$ normal; $1=$ mild; $2=$ moderate; and $3=$ severe) for both the stress and rest images. From this analysis, ischemia was defined as a change in segmental score between stress and rest. Segments with no change between stress and rest were classified as nonreversible. Summed stress and rest scores were calculated by summing the 17 segmental scores in each imaged set. Utilizing the summed difference score (SDS) in measuring defect reversibility was calculated from the difference between the summed stress and rest scores. A SDS lower than 2 was considered nonischemic, 2 to 7 was considered as mild ischemia, and greater than 7 as considered as moderate to severe ischemia. The reader made the final determination of an abnormal SPECT study by comparing both the perfusion and functional data. The perfusion defects represented by the perfusion scores at stress and rest were used to form the interpretation of the MPS studies. A gated SPECT result was considered normal if as follows: no visual perfusion defect, summed stress score $<3$, a left ventricular ejection fraction (LVEF) at rest $>50 \%$.

\section{Coronary artery calcium score acquisition and analysis}

All patients with heart rates $>70 \mathrm{bpm}$ received oral $\beta$-blocker therapy, with 50 or $100 \mathrm{mg}$ of metoprolol tartrate (AstraZeneca, Zoetermeer, the Netherlands) after stress testing to achieve a heart rate $<70 \mathrm{bpm}$ for the CAC scan. A non-enhanced CT scan (High Definition CT XT; GE Healthcare) during breathhold at end expiration to calculate the total CAC score was acquired with ECG triggering at $75 \%$ of the R-R interval and with the following scanning parameters: 40 or 48 sections and 2.5-mm section thickness; gantry rotation time, $330 \mathrm{~ms}$; tube voltage, $120 \mathrm{kV}$; and a tube current of $125 \mathrm{~mA}$. If patients were not able to hold their breath, a free breathing CT was acquired. Post processing was conducted at a dedicated workstation using Smartscore software (GE Healthcare). The CAC score was calculated using the standard Agatston criteria [5].

\section{Coronary CT angiography acquisition}

Patients without contraindications received metoprolol targeting a heart rate of $\leq 65 \mathrm{bpm}$ and nitroglycerin $0.8 \mathrm{mg}$ sublingually before image acquisition. A bolus tracking technique was used to calculate the time interval between intravenous contrast (Visipaque 320, GE Healthcare, Milwaukee, Wisconsin, USA) infusion and image acquisition. Final images were acquired with a triphasic protocol (100\% contrast, 40\%/60\% contrast/saline, and $40 \mathrm{~mL}$ saline). The contrast volume and infusion rate ( 5 to $6 \mathrm{~mL} / \mathrm{s}$ ) were individualized according to scan time and patient body habitus. Retrospective ECG-gated data sets were acquired with the GE high definition CT (GE Healthcare, Milwaukee, Wisconsin, USA) with $64 \times 0.625 \mathrm{~mm}$ slice collimation and a gantry rotation of $350 \mathrm{~ms}(\mathrm{~mA}=300$ to $800, \mathrm{kV}=120)$. Pitch $(0.16$ to 0.24$)$ was individualized to the patient's heart rate. The CCTA data sets were reconstructed with an increment of $0.4 \mathrm{~mm}$ using the cardiac phase with the 
Table 1. Baseline Demographics of The study Population

\begin{tabular}{ll}
\hline $\mathrm{N}$ & 157 \\
Age (years) & $53 \pm 10$ \\
Female & $88(56 \%)$ \\
Male & $69(44 \%)$ \\
Normal MPS & $122(78 \%)$ \\
Abnormal MPS & $35(22 \%)$ \\
Diabetes mellitus & $89(57 \%)$ \\
Hypertension & $66(66 \%)$ \\
Smoking & $14(9 \%)$ \\
Hypercholesterolemia & $33(33 \%)$ \\
Family history & $6(4 \%)$ \\
Left ventricular function & $60 \pm 11 \%$ \\
\hline
\end{tabular}

least cardiac motion.

\section{Coronary CT angiography image analysis}

Images were processed using the GE Advantage Volume Share Workstation (GE Healthcare, Milwaukee, Wisconsin, USA) and visually interpreted by two expert observers blinded to all clinical data. A 17-segment model of the coronary arteries and 4-point grading score (normal, mild $(<50 \%)$, moderate $(50 \%$ to $69 \%)$, severe $(\geq 70 \%))$ was used for the evaluation of coronary stenosis. Obstructive CAD was defined as coronary diameter stenosis $\geq 50 \%$ [15].

\section{Statistical analysis}

Data were entered into SPSS V 20.0 for analysis (SPSS, Chicago, Illinois). All continuous variables were expressed as mean \pm standard deviation (SD). Relationship between categorical variables was assessed using Chi-square test. Comparison between groups was performed using two independent sample $t$-test. A P value of less than 0.05 indicated statistically significant difference.

\section{Results}

Our sample consisted of 157 patients, (mean age: $53 \pm 10$ years,

Table 2. MPS Results

\begin{tabular}{ll}
\hline N & 157 \\
Normal MPS & $122(78 \%)$ \\
Abnormal MPS & $35(22 \%)$ \\
Fixed defect & $21(13 \%)$ \\
Equivocal & $10(6 \%)$ \\
Reversible defect & $4(3 \%)$ \\
\hline
\end{tabular}

Table 3. CT Coronary Angiography Results

\begin{tabular}{ll}
\hline $\mathrm{N}$ & 35 \\
Normal coronary artery & $30(85 \%)$ \\
Coronary artery stenosis & \\
$\quad$ More than $50 \%$ & $1(3 \%)$ \\
$\quad$ Less than $50 \%$ & $1(3 \%)$ \\
Anomalous coronary artery origin & $1(3 \%)$ \\
Myocardial bridging & $2(6 \%)$ \\
\hline
\end{tabular}

$88(56 \%)$ female patients, 69 (44\%) male patients) who were suspected to have CAD and having low to intermediate pretest likelihood for CAD according to the Diamond and Forrester criteria (Table 1). All patients had zero CACS. A total of 122 (78\%) patients had normal MPS, and $35(22 \%)$ had abnormal MPS, abnormal MPS includes fixed defect 22(13\%), equivocal $10(6 \%)$, and reversible defect $4(3 \%)$ (Table 2). All patients with abnormal MPS had further imaging with CTCA, CTCA was normal in $30(85 \%)$ patients, one patient had coronary artery stenosis more than $50 \%$, one patient had coronary artery stenosis less than $50 \%$, one patient had anomalous origin coronary artery, and two patients had myocardial bridging (Table 3 ). There were several findings in patients with abnormal MPS and normal coronary artery such as dilated cardiomyopathy in $14(40 \%)$ patients (Fig. 1a, b), asymmetrical septal hypotrophy in one $(3 \%)$ patient and mitral valve disease in three $(9 \%)$ patients (Table 4). There was no association with conventional CAD risk factors with MPS results.

\section{Discussion}

Our data show that a CAC score of zero has less than $1 \%$ $(0.6 \%)$ flow limiting CAD based on CCTA in symptomatic stable patients with low to intermediate probability of CAD. It appears that CAC score in this group of patients is appropriate, safe, and cost-effective as initial diagnostic test for CAD. However, the prevalence of abnormal MPS in patients with CAC score of zero is relatively high up to $22 \%$. Most patients with abnormal MPS do not have obstructive CAD. Based in our data, one patient out of 35 patients with abnormal MPS had obstructive CAD based on CCTA. Importantly, CCTA showed other findings that potentially explaining the abnormal perfusion on myocardial imaging such as dilated and hypertrophic cardiomyopathy, mitral valve disease, and myocardial bridging. This finding is extremely important indicating that not all abnormal myocardial perfusion in absence of obstructive CAD based on conventional coronary angiography is false positive.

According to our data, it is appropriate to initiate the cardiac workup for patients with low to intermediate risk with CAC score due to its relatively low cost, low radiation, and wide availability. Further functional imaging with MPS is indicated to exclude myocardial ischemia. In patients with zero CAC score and normal MPS no further workup is indicated, however, with abnormal MPS additional imaging with CCTA 

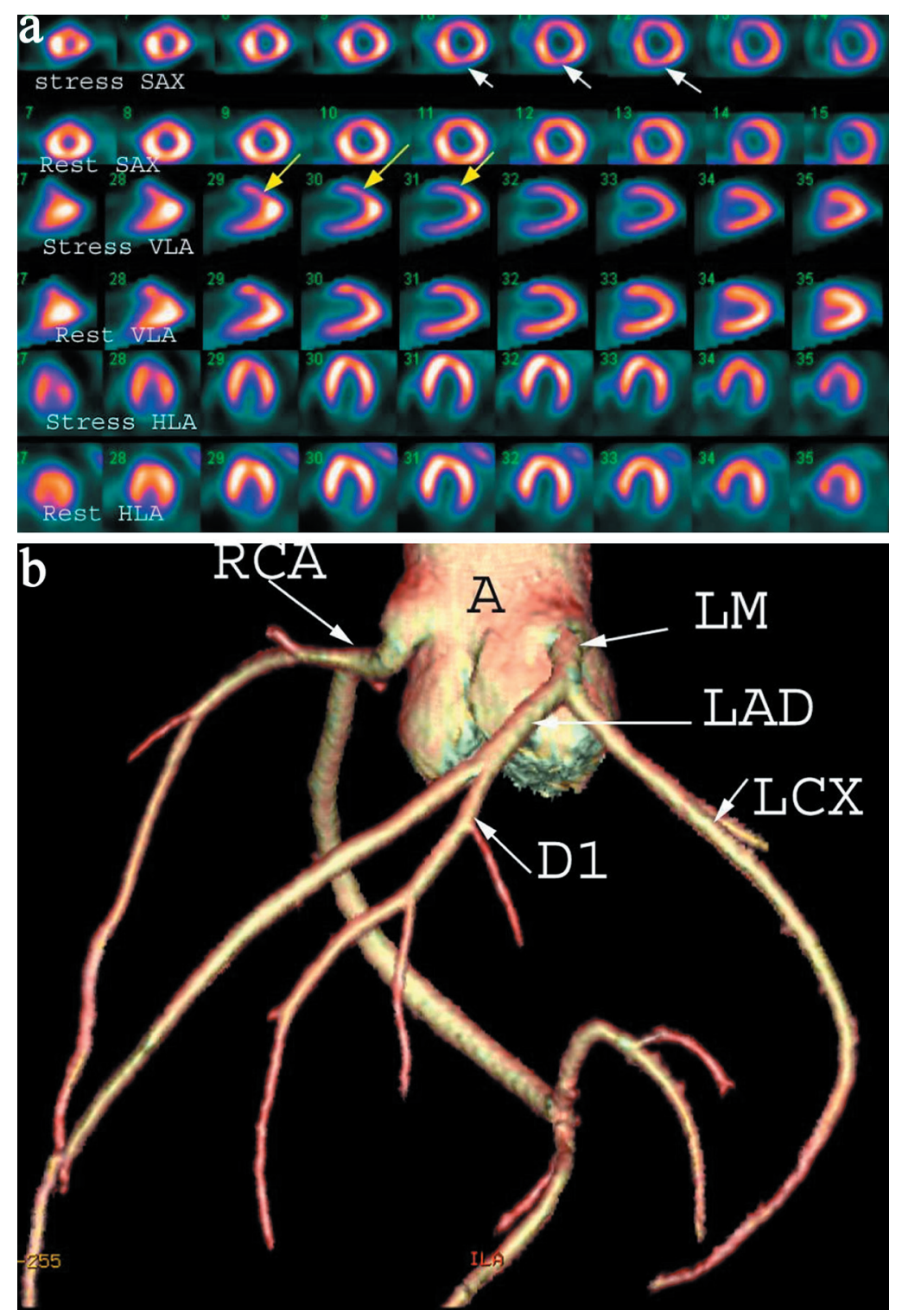

Figure 1. (a) A 42-year-old female with chest pain and shortness of breath. Myocardial perfusion scintigraphy demonstrates reversible defects in the apex and anterior wall (yellow arrows) and inferior wall (white arrows) suggesting inducible ischemia. Gated images (not shown) demonstrate mildly dilated left ventricular cavity and global hypokinesis and impaired left ventricle ejection fraction of 35\%. SAX: short axis; VLA: vertical long axis; HLA: horizontal long axis. (b) Shaded surface display coronary computed tomography angiogram demonstrating normal coronary arteries without calcified or non-calcified plaques. A: ascending aorta; LM: left main; LAD: left anterior descending artery; D1: first diagonal artery; LCX: left circumflex artery; RCA: right coronary artery.

may be indicated.

\section{Prevalence of obstructive CAD among patients with a zero CAC score}

The prevalence of obstructive CAD in patients with zero CAC is variable from less than $1 \%$ to about $3 \%$ based strongly on population studied, patient's presentation, and pretest probability of CAD. In asymptomatic patients with a zero CAC score, the prevalence of obstructive CAD is lower than $1 \%$
[16]. However, in symptomatic patients, a zero CAC score is associated with a low likelihood of up to $3 \%$. In a systematic review by Sarawar et al, it has been shown that absence of CAC was associated with a very low risk of further cardiovascular event; with a modest incremental value of other diagnostic test in this this very low risk group [17]. In another major study by Villines et al, 10,037 symptomatic patients without CAD who underwent CCTA and CAC scoring, of which $84 \%$ had no $\mathrm{CAD}, 13 \%$ had no obstructive $\mathrm{CAD}$, and $3.5 \%$ had more than $50 \%$ stenosis. It was concluded that in symptomatic patients with a zero CAC score, obstructive CAD is possible and is as- 
Table 4. CT Coronary Angiography and Cardiac Morphology Findings in Patients With Abnormal MPS

\begin{tabular}{ll}
\hline N & 35 \\
Normal CT coronary angiography and normal cardiac morphology & $13(37 \%)$ \\
Abnormal CT coronary angiography and/or abnormal cardiac morphology & $22(63 \%)$ \\
Dilated cardiomyopathy & $14(40 \%)$ \\
Coronary artery stenosis, more than 50\% & $1(3 \%)$ \\
Asymmetrical septal hypertrophy & $1(3 \%)$ \\
Myocardial bridging & $2(6 \%)$ \\
Anomalous coronary artery origin & $1(3 \%)$ \\
Mitral valve disease (stenosis, post mitral valve replacement) & $3(9 \%)$ \\
\hline
\end{tabular}

sociated with increased cardiovascular events, and CAC score did not add incremental prognostic information to CCTA [7].

\section{Relationship between zero CAC score and myocardial per- fusion}

In our study, 35 (22\%) patients out of 157 patients with zero CAC score have abnormal myocardial perfusion (abnormal MPS). The pattern of perfusion abnormalities includes fixed defect $21(13 \%)$, equivocal myocardial perfusion10 $(6 \%)$, and reversible defect $4(3 \%)$, but obstructive CAD is identified in one patient only based on CCTA. Interestingly, there are many cardiac morphological abnormalities on CCTA that could potentially explain the abnormal myocardial perfusion in absence of obstructive CAD. For example, CCTA shows one patient with anomalous origin right coronary artery from left coronary ostia, two patients with myocardial bridging, and one patient with non-obstructive CAD (less than $50 \%$ luminal stenosis). All these patients have zero CAC score, abnormal myocardial perfusion, and patent coronary arteries. This finding could explain patients' symptoms and abnormal myocardial perfusion and not necessarily indicate false positive MPS. In addition, there are several cardiac morphological abnormalities on CCTA, such as dilated cardiomyopathy (DCMP), hypertrophic cardiomyopathy (HCMP), and mitral valve disease. Previous study showed that, SPECT perfusion abnormalities in patients with normal coronary artery on CCTA was $12 \%$ [18] which is relatively less than our population. We strongly believe these important findings and may explain why in many previous studies that MPS had low specificity when compared to gold standard conventional coronary angiography. For example, in our patients, there are 14 patients out of 35 have DCMP, one patient has HCMP, and two patients has mitral valve disease. There are several possible reasons for the presence of perfusion defect in DCMP. The presence of myocardial fibrosis and scarring could account for fixed perfusion defect [19]. In addition, dilatation of left ventricle and abnormal cell membrane could cause variable tracer uptake and distribution. It was also known that stressinduced left ventricle geometric change may cause reversible perfusion defects in DCMP. Exercise-induced coronary spasm, mitral valve prolapse, and aortic valve stenosis had been associated with myocardial perfusion abnormalities
[20]. In hypertrophic cardiomyopathy, there is thickening of the myocardium, practically the septum; this can result in significant increased activity in the septum, which results in the significant deceased uptake in the other myocardial walls creating perfusion abnormalities [21].

\section{The negative predictive value of zero CAC versus exercise} testing and myocardial perfusion imaging

Dedic et al, in a study consistent of 791 consecutive stable patients showed that patients with stable chest pain who underwent CAC scoring and exercise electrocardiogram, a zero CAC score had substantial lower likelihood ratio compared to the exercise electrocardiogram; $0.11,0.13,0.13$ vs. $0.93,0.55$, 0.46 in the low, intermediate, and high risk group. In low risk patients $(0-30 \%)$ a negative CAC reduced the likelihood of obstructive CAD to $5 \%$ obviating the need for further diagnostic workup in substantial proportion of patients. For intermediate risk patients $(30-70 \%)$ the likelihood decreased to $5-25 \%$, but in high risk group zero CAC score cannot exclude obstructive CAD [22]. The negative predictive value of zero CAC score for detection of myocardial ischemia was investigated by Esteves et al. A total of 99 patients out of 206 patients had zero CACS, and of those 99 patients only one patient had myocardial ischemia on myocardial perfusion PET, also CACS of zero was the strongest independent factors of normal myocardial perfusion PET [8]. Despite this high negative predictive value of zero CACS for prediction of myocardial ischemia, we strongly believe, further testing with MPS or CCTA is necessarily in patients with zero CACS presented to the emergency department, patients with higher pretest probability, and young patients with high suspicious of coronary artery anomalies. CCTA is particularly helpful in patients with high suspicious of false positive MPS such as patients with DCMP, HCMP, left bundle branch block, coronary artery anomalous, and patients with known structural or congenital heart disease.

\section{Study limitations}

One of our study limitations is that our gold standard for evaluating CAD was CCTA instead of invasive coronary angiography, which is the gold standard method of detection of CAD, 
However, many studies have shown that CCTA has 90-94\% sensitivity and 96-100\% negative predictive value [23].Our patients with normal MPS did not undergo any further imaging, potential false negative could not be completely excluded due to many reasons such as balanced ischemia and or left main coronary artery disease. Also this group of patients with normal SPECT may have noncalcified plaque or non-obstructive CAD.

\section{Conclusions}

In conclusion, zero CACS in stable patients with low or intermediate risk indicates very low likelihood of obstructive CAD, less than $1 \%$ and normal MPS most likely will not benefit from further testing. However, patients with abnormal MPS will need further imaging with CCTA. CCTA is particular helpful in this group of patients for not only for evaluation of coronary artery disease but also for cardiac morphological evaluation.

\section{References}

1. Budoff MJ, Shaw LJ, Liu ST, Weinstein SR, Mosler TP, Tseng PH, Flores FR, et al. Long-term prognosis associated with coronary calcification: observations from a registry of 25,253 patients. J Am Coll Cardiol. 2007;49(18):1860-1870.

2. Simons DB, Schwartz RS, Edwards WD, Sheedy PF, Breen JF, Rumberger JA. Noninvasive definition of anatomic coronary artery disease by ultrafast computed tomographic scanning: a quantitative pathologic comparison study. J Am Coll Cardiol. 1992;20(5):1118-1126.

3. Detrano R, Guerci AD, Carr JJ, Bild DE, Burke G, Folsom $\mathrm{AR}$, Liu K, et al. Coronary calcium as a predictor of coronary events in four racial or ethnic groups. N Engl J Med. 2008;358(13):1336-1345.

4. Rozanski A, Gransar H, Wong ND, Shaw LJ, MirandaPeats R, Polk D, Hayes SW, et al. Clinical outcomes after both coronary calcium scanning and exercise myocardial perfusion scintigraphy. J Am Coll Cardiol. 2007;49(12):1352-1361.

5. Agatston AS, Janowitz WR, Hildner FJ, Zusmer NR, Viamonte M, Jr., Detrano R. Quantification of coronary artery calcium using ultrafast computed tomography. J Am Coll Cardiol. 1990;15(4):827-832.

6. Shaw LJ, Giambrone AE, Blaha MJ, Knapper JT, Berman DS, Bellam N, Quyyumi A, et al. Long-term prognosis after coronary artery calcification testing in asymptomatic patients: a cohort study. Ann Intern Med. 2015;163(1):1421.

7. Villines TC, Hulten EA, Shaw LJ, Goyal M, Dunning A, Achenbach S, Al-Mallah M, et al. Prevalence and severity of coronary artery disease and adverse events among symptomatic patients with coronary artery calcification scores of zero undergoing coronary computed tomography angiography: results from the CONFIRM (Coronary CT Angiography Evaluation for Clinical Outcomes: An International Multicenter) registry. J Am Coll Cardiol.
2011;58(24):2533-2540.

8. Esteves FP, Khan A, Correia LC, Nye JA, Halkar RK, Schuster DM, Stillman A, et al. Absent coronary artery calcium excludes inducible myocardial ischemia on computed tomography/positron emission tomography. Int J Cardiol. 2011;147(3):424-427.

9. Gottlieb I, Miller JM, Arbab-Zadeh A, Dewey M, Clouse ME, Sara L, Niinuma H, et al. The absence of coronary calcification does not exclude obstructive coronary artery disease or the need for revascularization in patients referred for conventional coronary angiography. J Am Coll Cardiol. 2010;55(7):627-634.

10. Haberl R, Tittus J, Bohme E, Czernik A, Richartz BM, Buck J, Steinbigler P. Multislice spiral computed tomographic angiography of coronary arteries in patients with suspected coronary artery disease: an effective filter before catheter angiography? Am Heart J. 2005;149(6):11121119.

11. van Werkhoven JM, de Boer SM, Schuijf JD, Cademartiri F, Maffei E, Jukema JW, Boogers MJ, et al. Impact of clinical presentation and pretest likelihood on the relation between calcium score and computed tomographic coronary angiography. Am J Cardiol. 2010;106(12):16751679.

12. Schenker MP, Dorbala S, Hong EC, Rybicki FJ, Hachamovitch R, Kwong RY, Di Carli MF. Interrelation of coronary calcification, myocardial ischemia, and outcomes in patients with intermediate likelihood of coronary artery disease: a combined positron emission tomography/computed tomography study. Circulation. 2008;117(13):16931700.

13. Diamond GA, Forrester JS. Analysis of probability as an aid in the clinical diagnosis of coronary-artery disease. N Engl J Med. 1979;300(24):1350-1358.

14. Henzlova MJ, Duvall WL, Einstein AJ, Travin MI, Verberne HJ. ASNC imaging guidelines for SPECT nuclear cardiology procedures: Stress, protocols, and tracers. J Nucl Cardiol. 2016;23(3):606-639.

15. Arbab-Zadeh A, Texter J, Ostbye KM, Kitagawa K, Brinker J, George RT, Miller JM, et al. Quantification of lumen stenoses with known dimensions by conventional angiography and computed tomography: implications of using conventional angiography as gold standard. Heart. 2010;96(17):1358-1363.

16. Yoon YE, Chang SA, Choi SI, Chun EJ, Cho YS, Youn TJ, Chung WY, et al. The absence of coronary artery calcification does not rule out the presence of significant coronary artery disease in Asian patients with acute chest pain. Int J Cardiovasc Imaging. 2012;28(2):389-398.

17. Sarwar A, Shaw LJ, Shapiro MD, Blankstein R, Hoffmann U, Cury RC, Abbara S, et al. Diagnostic and prognostic value of absence of coronary artery calcification. JACC Cardiovasc Imaging. 2009;2(6):675-688.

18. Mouden M, Timmer JR, Reiffers S, Oostdijk AH, Knollema S, Ottervanger JP, Jager PL. Coronary artery calcium scoring to exclude flow-limiting coronary artery disease in symptomatic stable patients at low or intermediate risk. Radiology. 2013;269(1):77-83.

19. Oakley C. Diagnosis and natural history of con- 
gested (dilated) cardiomyopathies. Postgrad Med J. 1978;54(633):440-450.

20. Bomb R, Kumar S, Chockalingam A. Coronary artery disease detection - limitations of stress testing in left ventricular dysfunction. World J Cardiol. 2017;9(4):304-311.

21. Burrell S, MacDonald A. Artifacts and pitfalls in myocardial perfusion imaging. J Nucl Med Technol. 2006;34(4):193-211; quiz 212-194.
22. Dedic A, Rossi A, Ten Kate GJ, Neefjes LA, Galema TW, Moelker A, van Domburg RT, et al. First-line evaluation of coronary artery disease with coronary calcium scanning or exercise electrocardiography. Int J Cardiol. 2013;163(2):190-195.

23. Stein PD, Yaekoub AY, Matta F, Sostman HD. 64-slice CT for diagnosis of coronary artery disease: a systematic review. Am J Med. 2008;121(8):715-725. 